\title{
Acute red ear in children: controlled trial of non-antibiotic treatment in general practice
}

\author{
Peter Burke, John Bain, Diane Robinson, Joan Dunleavey
}

Abstract

Objective-To examine the efficacy and safety of conservative management of mild otitis media ("the acute red ear") in children.

Design-Double blind placebo controlled trial.

Setting-17 group general practices (48 general practitioners) in Southampton, Bristol, and Portsmouth.

Patients - 232 children aged 3-10 years with acute earache and at least one abnormal eardrum (114 allocated to receive antibiotic, 118 placebo).

Interventions-Amoxycillin $125 \mathrm{mg}$ three times a day for seven days or matching placebo; $100 \mathrm{ml}$ paracetamol $120 \mathrm{mg} / 5 \mathrm{ml}$.

Main outcome measures-Diary records of pain and crying, use of analgesic, eardrum signs, failure of treatment, tympanometry at one and three months, recurrence rate, and ear, nose, and throat referral rate over one year.

Results-Treatment failure was eight times more likely in the placebo than the antibiotic group (14.4\% $v 1.7 \%$, odds ratio $8.21,95 \%$ confidence interval 1.94 to $34 \cdot 7$ ). Children in the placebo group showed a significantly higher incidence of fever on the day after entry $(20 \% v 8 \%, \mathrm{p}<0.05)$, mean analgesic consumption $(0.36 \mathrm{ml} / \mathrm{h} v 0.21 \mathrm{ml} / \mathrm{h}$, difference 0.14 , $95 \%$ confidence interval 0.07 to $0.23 ; p=0.0022$ ), mean duration of crying ( 1.44 days $v 0.50$ days, 0.94 0.50 to $1.38 ; p<0.001$ ), and mean absence from school $(1.96$ days $v 0.52$ days, $1.45 ; 0.46$ to 2.42 ; $p=0.0132$ ). Differences in recorded pain were not significant.

The prevalence of middle ear effusion at one or three months, as defined by tympanometry, was not significantly different, nor was there any difference in recurrence rate or in ear, nose, and throat referral rate in the follow up year.

No characteristics could be identified which predicted an adverse outcome.

Conclusions-Use of antibiotic improves short term outcome substantially and therefore continues to be an appropriate management policy.

Primary Medical Care Group, University of Southampton, Aldermoor Health Centre,

Southampton SO1 6ST Peter Burke, MRCGP, senior lecturer

John Bain, MD, professor Diane Robinson, BA, research officer

Joan Dunleavey, BED, research officer

Correspondence to: Dr P Burke, St Bartholomew's Medical Centre, Manzil Way, Oxford OX4 1XB

BMJ 1991:303:558-62
Negative results have come from a range of open trials $^{8-10}$ and also from two major controlled studies. Van Buchem et al found no difference in outcome among 171 children aged between 2 and 12 years given amoxycillin and placebo." In a subsequent study spontaneous resolution occurred in $90 \%$ of 1860 children aged between 2 and 12 treated initially without antibiotics. ${ }^{12}$ No children suffered long term illness as a result of mastoiditis (two cases), meningitis, or brain abscess. The second study, by Mygind et al, involving 149 children, also gave largely negative results. ${ }^{13}$

Meanwhile, routine antibiotic treatment has been suggested to be associated with incomplete resolution and to have contributed to the observed rise in the prevalence of effusion of the middle ear. ${ }^{14}$ is In view of this it is increasingly recommended that use of antibiotics should be selective, being confined to those children who are severely affected - for example, those who have bulging eardrums - a physical sign which predicts bacterial infection.

The costs and benefits of this recommendation remain uncertain. This study is the first randomised trial of non-antibiotic treatment of acute earache to be carried out in British general practice. We decided to compare this treatment with the most traditional regimen-namely, a seven day course of amoxycillin, to test the hypothesis that outcome in children treated conservatively for acute earache is similar to that with a conventional course of antibiotics.

\section{Patients and methods}

The study was approved by the Southampton and District Ethical Committee. An assessment of sample size suggested that some 200 patients would need to be recruited, based on the need to identify a difference between $15 \%$ and $30 \%$ in any given outcome measure with a power of $0 \cdot 80$.

A total of 48 general practitioners in 17 practices in Southampton, Bristol, and Portsmouth took part. Patients were recruited during the winter months (October to April) of 1986-7, 1987-8, and 1988-9.

We held workshops to explain the aims and methods of the study and agree the descriptive terms, with the help of standard sets of slides depicting a range of common eardrum appearances, and the participating doctors were given a set of sample photographs to help them standardise terminology.

We invited general practitioners to consider for inclusion children aged at least 3 years and less than 10 years who presented with acute earache and had at least one abnormal eardrum. Children who had had antibiotic treatment or otitis media in the preceding two weeks, those for whom antibiotics were thought to be strongly indicated, those known to be allergic to penicillins, and those with serious chronic disorders were excluded. Children were not included if they had been entered into the study within the previous 12 months. 
FIG 1-Physical signs recorded at entry to trial

\section{Ear \\ A Tympanic membrane adequately seen \\ B Tympanic membrane normal

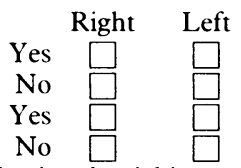 \\ If "no," please complete the following, by ticking on} box in each section. NB "Normal" appears at the beginning of each section.
C Colour
Normal colour
Red rim only
Injection of malleus/rim only
Red membrane $(<2 / 3)$ Red membrane $(>2 / 3)$
D Contour Normal Bulging Indrawn
E Light reflex Present
F Fluid level or bubbles: Absent
G Perforation Noperforation Pus seen, perforation assumed Perforation seen

At first presentation (visit 1 , day 1 ) the general practitioner obtained informed parental consent; completed a record of physical signs in the ears (fig 1); and recorded relevant symptoms and their duration with particular reference to earache, ear discharge, and respiratory or gastrointestinal symptoms. Parents were given a diary, in which they recorded these symptoms four hourly for 24 hours. A bottle containing either a course of amoxycillin $125 \mathrm{mg}$ three times a day for seven days or matching placebo was given. Each bottle was identified only by number, and randomisation was carried out independently of the investigators with computer generated random numbers. The randomisation code was kept sealed and was unknown to any of the participants in the study. A second bottle, containing $100 \mathrm{ml}$ paracetamol $120 \mathrm{mg} / 5 \mathrm{ml}$ (Disprol) was also given.

On the day after entry (visit 2) a researcher visited the child at home, obtained further historical information, asked about current pain, measured the child's temperature, weighed the medicine bottles, and replaced the 24 hour diary with a 21 day diary, to be completed daily by the parent. This diary required entries for aural, respiratory, and gastrointestinal symptoms and, in children over 5 years old, absence from school.

Each child was reviewed at home by the researcher on days 5-7 (visit 3), and in the surgery by the general practitioner on day 8 (visit 4). (An additional visit to the general practitioner on day 3 was optional.) General practitioners were free to withdraw children from the study at any time and to prescribe a second line antibiotic (co-trimoxazole and amoxycillin/clavulanate were recommended) in the event of apparent failure of treatment. When this occurred the randomisation code was not broken (though there was provision to do so in an emergency) and the child was followed up according to the protocol.

The principal outcome measures are given below:

Short term-(a) Duration of symptoms; (b) use of analgesics (assessed by weighing of bottles); (c) clinical signs at one week; $(d)$ incidence of complications; $(e)$ failure of treatment - that is, whether a second line antibiotic was required.

Medium term-Findings on tympanometry with an earscan II (Micro-Audiometrics) at one and three months, the tympanograms being classified with a method modified from Jerger. ${ }^{16}$

Long term-(a) Number of episodes of otitis media in the subsequent year; $(b)$ whether specialist referral was required.
Data were analysed with the statistical package for the social sciences (SPSS) $\left(\chi^{2}\right.$ and Mann-Whitney U tests), and confidence intervals ${ }^{17}$ were calculated. The principal analysis was on an intention to treat (pragmatic) basis. When appropriate, stratification was used. The randomisation codes were not broken until one year after entry to the trial.

\section{Results}

A total of 232 eligible children were entered into the study, 114 in the antibiotic group and 118 in the placebo group. Reliable information about patients excluded from the study was not obtainable for all participating practices. However, in one practice a policy of systematically recording such patients was followed. Eighty four patients were recruited and 105 met the entry criteria but were excluded (inferred recruitment rate $44 \%$ ). The reasons for exclusion were an indication for antibiotic (52 children: bulging eardrum (27), perforation or pus (19), severe illness (five), and grommets (one)); previous recruitment in preceding year (15); lack of time (eight); absence of parental consent (six); antibiotic treatment in preceding two weeks (five); allergy to penicillin (four); concomitant medical conditions (three); eardrum inadequately seen (four); reason not stated (eight).

\section{CHARACTERISTICS OF CHILDREN}

Boys were slightly more common in the antibiotic group $(59 / 114,52 \%)$ than in the placebo group $(50 / 118$, $42 \%$ ), but the groups did not differ in terms of age structure, geographical location, social class, season of entry, recorded history of otitis media, and history of ear, nose, and throat referral or ear surgery. Four children receiving antibiotic and eight placebo had had an adenoidectomy. Mean duration of pain before entry to the trial was $35 \cdot 8$ hours in the antibiotic group and $30 \cdot 1$ hours in the placebo group (difference not significant). The two groups had similar physical signs at entry, except that 19 children had one or more bulging eardrums in the antibiotic group compared with eight in the placebo group.

By visit 3, 89 children (78\%) in the antibiotic group and $82(69 \%)$ in the placebo group had taken at least two thirds of the appropriate amount of their treatment. Though data for some individual children were incomplete, the children were included in any analysis for which they were eligible. For this reason the denominators varied.

Completed diaries were returned for 220 children; 228 were available for tympanometry at one month and 222 at three months. Table I summarises the principal findings.

\section{SHORT TERM OUTCOME}

Figure 2 shows the duration of crying and pain based on the diaries of symptoms, and the last consecutive day at which each was present. There was a significant difference in duration of crying between the two groups; children in the antibiotic group ceased crying earlier than those in the placebo group (mean time to cessation of crying 0.5 days $v 1.44$ days respectively; $p<0 \cdot 0001$, Mann-Whitney $U$ test). Note that as figure 1 is a survival curve, the differences between the groups at time 0 represent a real difference in outcome, rather than in characteristic, at entry. On the other hand, there was no significant difference in the children reporting pain at either home visit (table I) nor in experience of pain recorded in the diaries. The mean time to cessation of pain was 2.82 days in the antibiotic group and 3.34 days in the placebo group $(p=0 \cdot 36)$. Ninety eight children in the antibiotic group and 102 in the placebo group reported unilateral pain before presentation. During the three weeks after 
TABLE I-Principal outcome measures in children with acute red ear randomised to receive amoxycillin or placebo

\begin{tabular}{|c|c|c|c|c|c|}
\hline & $\begin{array}{l}\text { Antibiotic } \\
\text { group }\end{array}$ & $\begin{array}{l}\text { Placebo } \\
\text { group }\end{array}$ & $\begin{array}{l}\text { Ratio placebo: } \\
\text { antibiotic }\end{array}$ & $\begin{array}{c}95 \% \text { Confidence } \\
\text { interval }\end{array}$ & $p$ Value \\
\hline \multicolumn{6}{|c|}{ Short term } \\
\hline No (\%) with: & & & & & \\
\hline Pain at visit 2 & $53 / 112(47)$ & $56 / 117(48)$ & 1.01 & 0.77 to 1.33 & $>0.05$ \\
\hline Fever at visit 2 & $7 / 87(8)$ & $19 / 93(20)$ & $2 \cdot 54$ & $1 \cdot 12$ to 5.74 & $<0 \cdot 05^{\star}$ \\
\hline Pain at visit 3 & $20 / 111(18)$ & $29 / 114(25)$ & $1 \cdot 41$ & 0.85 to 2.34 & $>0.05$ \\
\hline Fever at visit 3 & $3 / 51(6)$ & $8 / 70(11)$ & 1.94 & 0.54 to 6.97 & $>0.05$ \\
\hline Worsening in eardrum signs & $15 / 103(15)$ & $26 / 110(24)$ & 1.62 & 0.91 to 2.89 & $>0.05$ \\
\hline Occurrence of discharging ear & $16 / 114(14)$ & $22 / 118(19)$ & $1 \cdot 33$ & $0 \cdot 74$ to $2 \cdot 40$ & $>0.05$ \\
\hline Contralateral pain & $29 / 98(30)$ & $33 / 102(32)$ & 1.09 & 0.72 to 1.66 & $>0.05$ \\
\hline Failure of treatment $\dagger$ & $2 / 114(2)$ & $17 / 118(14)$ & $8 \cdot 21$ & 1.94 to $34 \cdot 7$ & $0.001^{\star}$ \\
\hline \multicolumn{6}{|l|}{ Mean (No): } \\
\hline Duration of pain (days) & $2 \cdot 82(107)$ & $3 \cdot 34(113)$ & 0.515 & -0.41 to 1.45 & $0 \cdot 36 \ddagger$ \\
\hline Duration of crying (days) & $0.50(106)$ & $1.44(113)$ & 0.945 & 0.50 to 1.38 & $<0.001 \neq$ \\
\hline $\begin{array}{l}\text { Analgesic consumption to } \\
\text { visit } 2(\mathrm{ml} / \mathrm{h})\end{array}$ & $0.72(14)$ & $0.77(118)$ & 0.050 & $0.27+0.0 .128$ & $0.59 \pm$ \\
\hline Analgesic consumption to & $0.72(114)$ & $0.7 /(118)$ & $0.05 y$ & -0.22100 .128 & $0.39+$ \\
\hline visit $3(\mathrm{ml} / \mathrm{h})$ & $0 \cdot 21(104)$ & $0 \cdot 36(111)$ & $0 \cdot 145$ & 0.07 to 0.23 & $0.0022 \ddagger$ \\
\hline $\begin{array}{l}\text { Days off school (children aged } \\
>5 \text { years) }\end{array}$ & $0.52(42)$ & $1.96(40)$ & $1.45 \mathrm{~S}$ & 0.46 to $2 \cdot 42$ & $0.0132 \ddagger$ \\
\hline \multicolumn{6}{|c|}{ Medium term } \\
\hline No (\%) with: & & & & & \\
\hline $\begin{array}{l}\text { Effusion in one or both ears at } \\
\text { one month }\end{array}$ & $41 / 112(37)$ & $41 / 116(35)$ & 0.97 & 0.68 to 1.37 & $>0.05$ \\
\hline $\begin{array}{l}\text { Effusion in one or both ears at } \\
\text { three months }\end{array}$ & $20 / 111(18)$ & $31 / 111(28)$ & 1.55 & 0.94 to 2.55 & $>0.05$ \\
\hline \multicolumn{6}{|c|}{ Long term } \\
\hline $\begin{array}{l}\text { No }(\%) \text { referred to ear, nose, } \\
\text { and throat }\end{array}$ & $7 / 112(6)$ & $9 / 111(8)$ & $1 \cdot 30$ & 0.50 to 3.36 & $>0.05$ \\
\hline Mean (No) recurrence rate & $0.69(112)$ & $0.63(111)$ & $-0.06 \$$ & -0.34 to 0.22 & NS \\
\hline
\end{tabular}

${ }^{\star} \gamma^{2}$ test.

†Non-resolution or recurrence of symptoms requiring use of second line antibiotic during first week.

$\ddagger M a n n-W h i t n e y ~ U$ test.

$\$$ Difference.
Table II shows that in 19 children a different antibiotic was started on or before day 8 because of non-resolution or recurrence of symptoms (failure of treatment), of whom 17 had been randomised to the placebo group and only two to the antibiotic group. The rate of treatment failure was $1.8 \%$ in the antibiotic group and $14.4 \%$ in the placebo group (odds ratio 8.21 ; $95 \%$ confidence interval 1.94 to $34 \cdot 7$ ). The rate was unaffected by age, history, or laterality of pain (table III). Three children were withdrawn from treatment because of presumed side effects: diarrhoea (one child) and rash (two children, one of whom was in the placebo group). During three weeks vomiting was reported for $20(18 \%)$ children in the antibiotic group and $14(12 \%)$ in the placebo group, diarrhoea for $24(21 \%)$ and 16 (14\%) respectively, and rash for nine $(8 \%)$ and six $(5 \%)$ respectively. (Symptoms present before trial entry were excluded.)

TABLE II-Withdrawal from treatment during trial. Figures are numbers of children

\begin{tabular}{|c|c|c|}
\hline & Antibiotic group & Placebo group \\
\hline \multicolumn{3}{|l|}{ Treatment failures: } \\
\hline Relapse & 2 & 2 \\
\hline Perforation & & 2 \\
\hline Other non-resolution & & 13 \\
\hline Parental initiative & 1 & 1 \\
\hline Associated features: & & \\
\hline Cough & 1 & \\
\hline \multicolumn{3}{|l|}{ Side effects: } \\
\hline Rash & 1 & 1 \\
\hline Diarrhoea & 1 & \\
\hline
\end{tabular}

entry to the trial the number of these children recorded as experiencing pain in the contralateral ear at any time was $29(30 \%)$ and $33(32 \%)$ respectively $(p>0.5)$.

Mean consumption of analgesic was similar between the groups at the time of visit 2 (day 2), but by the time of visit 3 (days 5-7) there was a significant excess among children treated without antibiotic (table I), which was sustained after correction for body weight and for interval between entry to the trial and the visit. Significantly more children in the placebo group had a fever at visit 2 , and this difference was sustained but non-significant by visit 3 (table I).

Of those children who presented for review by the general practitioner for visit 4 (day 8), 15/103 (15\%) in the antibiotic group and $26 / 110(24 \%)$ in the placebo group showed clear evidence of clinical deterioration in one or both ears. This difference was not significant $\left(\chi^{2}=2 \cdot 26, \mathrm{df}=2, \mathrm{p}>0 \cdot 05\right)$. (These figures include two children in the antibiotic group and four in the placebo group who showed bilateral deterioration of the ears.)

The mean duration of absence from school (excluding the day of entry) recorded in children aged 6 or more (except for 1986-7 and during school holidays) was significantly longer in the placebo group $(1.96$ placebo group $v 0.52$ antibiotic group; $\mathrm{p}=0.013$; table I).

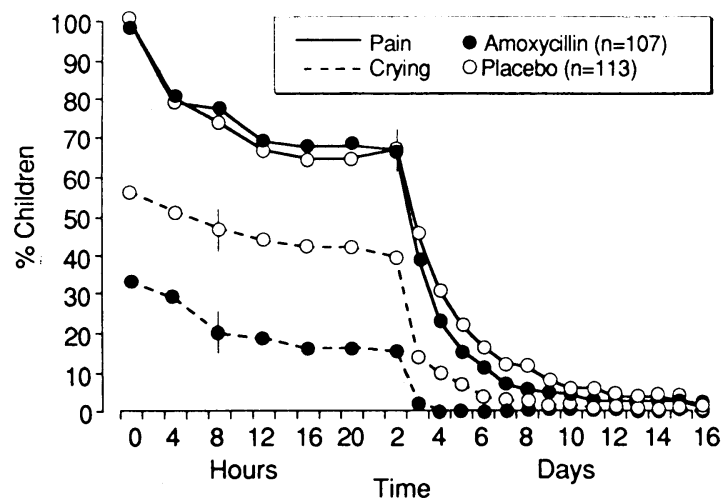

FIG 2-Mean time to cessation of pain and crying. Percentages represent those children whose initial episode had persisted up to or beyond that time, therefore data for crying represent true differences in outcome rather than in entry characteristics (denominator = all children for whom diaries had been completed); bars are $95 \%$ confidence intervals

TABLE III-Failure of treatment in children with acute red ear allocated to receiving placebo, by age, previous episodes, and presentation

\begin{tabular}{lr}
\hline Variable & No $(\%)$ \\
\hline Age $($ years $):$ & \\
$3-5(n=66)$ & $10(15)$ \\
$6-9(n=52)$ & $7(13)$ \\
Previous episodes: & $6(11)$ \\
$0-2(n=55)$ & $7(15)$ \\
$>2(n=48)$ & \\
Presentation: & $14(14)$ \\
Unilateral $(n=102)$ & $3(19)$ \\
Bilateral $(n=16)$ & \\
\hline
\end{tabular}

\section{MEDIUM TERM OUTCOME}

Tympanometry was carried out at one month and three months in 228 and 212 children respectively. Figure 3 shows the results, classified by findings in the worst ear. The groups were similar at one month. At three months there was an excess of children with effusions in the placebo group ( $31 v 20)$, and this was accounted for largely by children with unilateral effusions (18 $v$ eight, $\chi^{2}=3 \cdot 53$ ). When the data were analysed by laterality of onset there was no difference among children with bilateral onset or at one month but among those with unilateral onset there were more effusions present in the placebo group in the ipsilateral ear at three months $(26 / 94,27 \cdot 6 \% v 11 / 95,11 \cdot 6 \%$; odds ratio $=2 \cdot 39 ; 95 \%$ confidence interval $1 \cdot 25$ to $4 \cdot 55$ ).

\section{LONG TERM OUTCOME}

Children's notes were reviewed one year after entry to the trial. Four children in the antibiotic group and seven in the placebo group had moved away or been lost to follow up. Of the remainder, $7 / 110(6 \%)$ in the antibiotic group and $9 / 111(8 \%)$ in the placebo group had been referred to an ear, nose, and throat outpatient clinic. The mean number of recorded recurrences of otitis media or acute red ear was 0.70 (range $0-4$ ) in the antibiotic group and 0.63 (range $0-7$ ) in the placebo group and this difference was not significant (difference $=0.06 ; 95 \%$ confidence interval -0.22 to $0.339)$.

None of the following variables were found to 


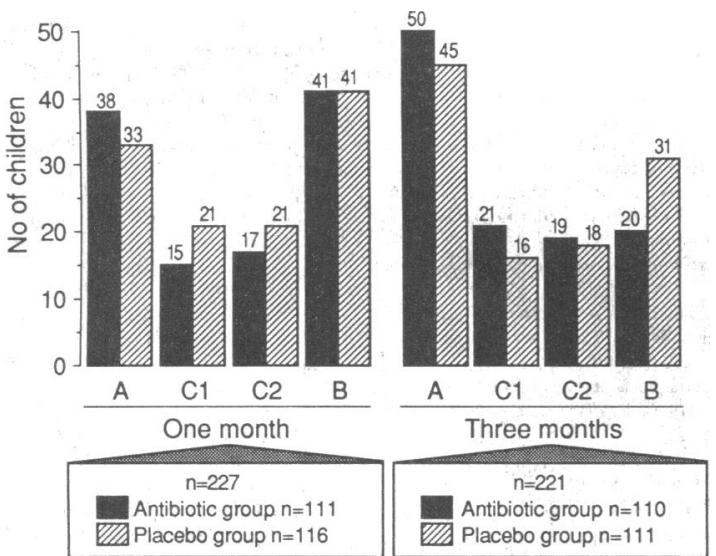

FIG 3-Tympanometric findings at one month and three months, classified according to the findings in the more severely affected ear. $(A$ normal, compliance $\geqslant 0.2 \mathrm{ml}$, middle ear pressure $>-1 \mathrm{kPa} ; \mathrm{Cl}$ middle ear pressure - 1 to $-2 \mathrm{kPa} ; \mathrm{C} 2$ middle ear pressure $<-2 \mathrm{kPa}$; $B$ flat tympanogram, compliance $<0 \cdot 2 \mathrm{ml}$ (highly predictive of effusion)). (Figures exclude one child with grommets)

influence the probability of treatment failure among the children in the placebo group: age, sex, social class, season, history of ear problems (grommets, number of previous episodes), laterality of onset, duration of pain, crying at onset, presence of cough, or appearance of eardrum.

A second, explanatory, analysis excluded those children in the antibiotic group who had taken less than two thirds of the appropriate quantity of prescribed treatment and all patients who had received secondary antibiotic treatment. The principal findings were similar in direction to those in the intention to treat analysis.

A total of 27 children with bulging eardrums were included in the study, and the data were reanalysed after excluding these children. The results were substantially similar to those given above. In particular, only one of the children in whom treatment failure subsequently occurred had presented with a bulging eardrum.

\section{Discussion}

This work set out to study the acute painful red ear, a syndrome presumed to be due to acute suppurative otitis media, although in general practice a precise diagnosis is not always possible.

The children included in the study did not represent a cross section of all those with acute earache but were selected on the basis that treatment with placebo would raise no ethical problems, and their inclusion was subject to informed parental consent. Excluded children may have been more severely affected, and this is supported by the records of one participating practice. The children in the study are typical, however, of those with moderate symptoms and signs, whose treatment presents general practitioners with a dilemma.

The number of children included had been estimated at the outset as sufficient for analysis of common complications, such as effusion of the middle ear, but it was known to be insufficient for analysing rarer events such as mastoiditis (incidence $<1 / 1000$ episodes $^{12}$ or meningitis. Neither of these conditions was observed in this study, and they are now sufficiently rare that fear of them does not compel the use of antibiotics. In our study there were slightly more girls than boys whereas published work-for example, that of Teele et $a l^{18}$-seems generally to describe a male predominance: this factor is, however, unlikely to influence the main findings.

The measures of short term outcome used favoured the children treated with antibiotic. This is particularly striking in view of the fact that this group may by chance have been more severely affected - the only difference between the groups at the outset was an excess of bulging eardrums among children treated with antibiotics.

A key finding of the study was that children treated with placebo were significantly more likely to require secondary antibiotic treatment. Our results are comparable with those recently published by Kaleida et al. ${ }^{19}$ By inference from our data, when antibiotics were withheld, about $12 \%$ of children experienced severe or persistent symptoms who would not otherwise have done so. It might be argued that most children in the placebo group showed satisfactory resolution of their symptoms and that antibiotics could reasonably be withheld for 24-48 hours; conversely, however, this seems to be possible only at the cost of increased suffering. Diary records of pain were discordant with other measures of short term outcome in this study, such as consumption of analgesics and crying. We suggest that such diaries are not a highly valid measure of outcome in children and this may help to account for the negative findings of previous studies. ${ }^{11} 13$

In terms of medium term outcome the only difference between the two groups was a slight reduction in the prevalence of effusion of the middle ear at three months in the antibiotic group. Our observations tend to refute the hypothesis that the use of antibiotics has contributed to the apparently increasing prevalence of middle ear effusion. ${ }^{14}$

In those children with unilateral presentation of acute red ear the occurrence of pain in the contralateral ear did not seem to be influenced by antibiotic treatment. In that ear the disease process may be at an earlier stage, and it has been speculated that intervention at this stage is likely to prevent progression. Our data do not support this hypothesis.

The number of children requiring referral was too small to allow any inferences to be drawn, but our negative findings in this respect seem consistent with those of others. Recurrence rates of earache seem not to be substantially influenced by treatment.

We accept the need for rigorous criteria of significance when multiple outcome measures are studied. However, the results of this study are internally consistent, and no single measure of outcome favours placebo treatment. Previous studies showed that antibiotics are as effective when given for two to three days as for the conventional seven to 10 days, ${ }^{20-22}$ and this is an approach which may be used when compliance with longer term treatment is considered a potential problem.

In summary, this study provides evidence that short term outcome of acute painful red ear is improved by antibiotic treatment but no evidence that medium and long term outcomes are affected. Given a child with an acute painful red ear, it is not possible from our results to define subgroups in whom antibiotics may be withheld. When deciding to withhold immediate antibiotic treatment it is particularly important to ensure early review.

We thank the Medical Research Council, The scientific foundation board of the Royal College of General Practitioners, Beecham Research, and Reckitt \& Colman for financial support; Mrs Patricia Byng and Miss Christine Slade for their help with data collection; Dr Michael Campbell and Dr Ann-Louise Kinmonth, University of Southampton, for valuable advice; and the general practitioners and their patients who took part in the study.

1 Mills RP. Policies on antibiotics of south east London general practitioners for managing acute otitis media in children. BMF 1984;288:1199-201.

2 Browning GG. Childhood otalgia: acute otitis media - antibiotics not necessary in most cases. BMF 1990;300:1005-6.

3 Bain DJG. Justification for antibiotic use in general practice. BMF 1990;300 1006-7. 
+ Laxdal OE, Merida J, Jones RHT. Treatment of acute otitis media: controlled study of $1+2$ children. Can Med Assoc f 1970;102:263-8.

5 Halsted C. Lepow MI. Balassanian N. Emmerich J, Wolinsky E. Otiti media: clinical observations, microbiology, and evaluation of therapy Am f Dis Child 1968:115:542-51.

6 Howie VM, Ploussard JH. Efficacy of fixed combination antibiotics versu separate components in otitis media. Clin Pediatr Phila) 1972:11:205-14.

7 Engelhard D, Strauss N, Jorczak-Sarni L, Cohen D, Sacks TG, Shapiro M Randomised study of myringotemy, amoxycillin/clavulanate, or both, for acute otitis media in infants. Lancet 1989;ii:141-3.

8 Fry J. Antibiotics in acute tonsillitis and acute otitis media. BMF 1958;ii: $883-6$

9 Medical Research Council. Acute otitis media in general practice-report of a surver by the Medical Research Council's working party for research in general practice Lance 1957:ii:510-t.

10 Froom J, Culpepper L, (irob P, et al. Diagnosis and antibiotic treatment of acute otitis media: report from International Primary Care Network. BMF
$1990 ; 300 \cdot 582-7$.

II Van Buchem FL, Dunk JHM, Van "T Hof MA. Therapy of acute otitis media: myringotoms, antibiotics or neither? Lancet 1981;ii: $883-7$.

2 Van Buchem FL, Peeters MF, Van 'T Hof MA. Acute otitis media: a new treatment strategy. B.M7 1985:290:1033-7.

13 Mvgind N, Meistrup-Larsen K-I, Thomsen J, Thomsen VF, Josefsson K, Sorensen H. Penicillin in acute otitis media: a double-blind placebocontrolled trial. Clin Otolaryngol 1981;6:5-13.
1+ Diamant $M$, Diamant $B$. Abuse and timing of use of antibiotics in acute otiti media. Arch Otolurvngol 1974;100:226-32.

15 Mills R. Uttley A, Mclntyre M. Relationship between acute suppurative otiti media and chronic secretory otitis media: role of antibiotics. $f \mathrm{R} \mathrm{Soc} \mathrm{Med}$ 1984;77:754-7.

16 Jerger J. Clinical experience with impedence audiometry. Arch Otolaryngol $1970 ; 92: 311-24$

17 Altman DG, Gardner MJ. Statistics with confidence. London: BMA 1989

18 Teele $\mathrm{DW}$, Klein JO, Rosner B, et al. Epidemiology of otitis media during the first seven years of life in children in greater Boston: a prospective cohor study. I Infect Dis 1989;160:83-94.

19 Kaleida PH, Casselbrandt ML, Rockette HE. Amoxycillin or mvringotomy or both for acute otitis media. Results of a randomized clinical trial. Pediatrics 1991;87:466-74.

20 Chaput de Saintonge DM, Levine DF, Temple Savage 1, et al. Trial of three-day and ten-day courses of amoxycillin in otitis media. B.MF 1982;284: $1078-81$.

21 Bain DJG, Murphy E, Ross F. Acute otitis media: clinical course amon children who received a short course of high dose antibiotic therapy. $B M Y$ children who recis

22 Jones $\mathrm{R}$. Bain J. Three-day and seven-day treatment in acute otitis media: double-blind antibiotic trial. $7 R$ Coll Gen Pract 1986;36:356-8.

(Accepted 17 May 1991)

\section{The Future of General Practice}

\section{Funding family health services}

David Taylor (box).

\section{King's Fund Institute,} London NW1 7NF

David Taylor, BSC, fellow in health policy analysis

$B M F 1991 ; 303: 562-4$
Building Your Own Future offers a refreshing break with the past. It raises many questions previously glossed over, and its publication offers hope that the period after the next general election could be one in which health care policies can be based more on reason and mutual agreement rather than dogma and conflict.

However, good intentions alone do not guarantee anything. A commonsense awareness of the resource restraints and differences of interest that affect the United Kingdom's health and social care system will always be needed to help generate a sound sense of the attainable. In this article I therefore briefly examine some of the broad economic factors which have influenced recent developments in general medical practice and will continue to affect the future of primary care in Britain. I do so in the context of questions suggested in Building Your Own Future

\section{Control of NHS expenditure, 1981-91}

From the start of the 1980s spending on the NHS has been maintained at about $6 \%$ of the gross national product (fig 1). Other areas of public expenditure, notably housing, have been more severely restricted, and the NHS has enjoyed real growth in its resources because of the overall expansion of the economy. Nevertheless, control of spending relative to national

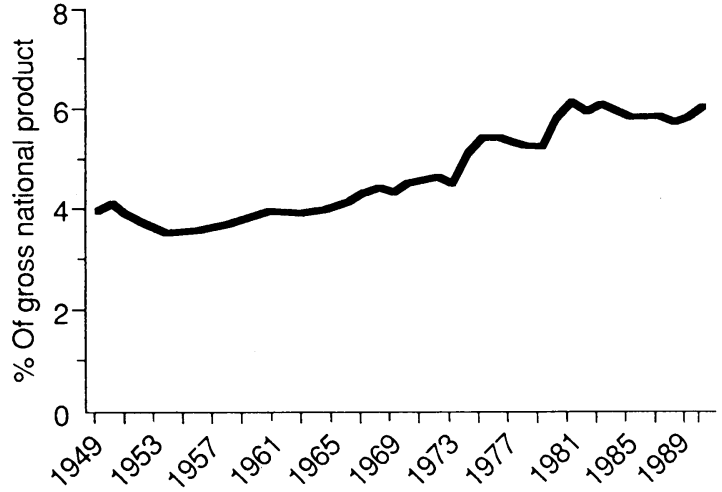

FIG 1-NHS expenditure as percentage of gross national product, United Kingdom 1949-90 ( $R$ Chew, personal communication)

wealth has clearly been an important goal of health policy during the past decade.

In the hospital and community health sector the cash limits system introduced during the 1970s helped to make strict cost containment possible. But the family health services retained a funding system based on a separate "open ended" parliamentary vote, ostensibly because of the need for flexibility to finance variable demands for items such as medicines. (Although family health services account for only $20 \%$ of total NHS costs, $80 \%$ of all NHS medicines by value are dispensed by family health services contractors.)

The treasury has always feared that family health

Financial questions raised in Building Your Own Future ${ }^{1}$

- Does the open nature of the general medical services prevent cash limits being applied?

- To what extent has the uncompromising opposition of the GMSC to cash limits led to presently unsatisfactory arrangements for applying cash limits on staff and premises spending?

- Should there in future be a single cash limit for the whole of NHS expenditure or separate cash limits for primary and secondary care?

- Does the GP fundholding model offer an appropriate vehicle for the future application of cash limits? compensatory cuts in the hospital and community sector or elsewhere. But general medical and pharmaceutical services in fact accounted for a relatively stable - and, in the case of the general medical services, falling - proportion of NHS costs during the 1960 s and 1970s (fig 2). Also, any overspend in such services is likely to be limited compared with overall government outlays. Nevertheless, the health departments were obliged to commission in 1982 the Binder Hamlyn study of family health service funding.

This exercise was followed by a cascade of events and 